\title{
Transverse modes of a bunched beam with space charge dominated impedance
}

\author{
V. Balbekov* \\ Fermi National Accelerator Laboratory, P.O. Box 500, Batavia, Illinois 60510, USA
}

(Received 20 August 2009; published 16 December 2009)

\begin{abstract}
Transverse coherent oscillations of a bunched beam in a ring accelerator are considered with space charge dominated impedance, taking into account linear synchrotron oscillations. A general equation of the bunch eigenmodes is derived, its exact analytical solution is presented for boxcar bunch, and numerical solutions are found for several realistic models. Both low and high synchrotron frequency approximations are considered and compared, fields of their applicability are determined, and some estimations are developed in the intermediate region. It is shown that most of the bunch eigenmodes are stabilized by Landau damping due to the space charge produced tune spread.
\end{abstract}

DOI: $10.1103 /$ PhysRevSTAB.12.124402

PACS numbers: 29.27.Bd

\section{INTRODUCTION}

Transverse instability of a bunched beam in a ring accelerator with synchrotron oscillations taken into account has been considered first by Pellegrini [1] and Sands [2]. Coherent oscillations of any bunch were presented as a sum of uncoupled modes $\propto \exp (\operatorname{im} \phi)$ where $\phi$ is synchrotron phase. After that, Sacherer [3] investigated this effect in depth including so-called radial modes which describe dependence of the displacement on synchrotron amplitude. Space charge effects have been examined first in Ref. [4] by analysis of the bunch eigenmodes with space charge dominated impedance. It was shown that most of the modes become stable due to Landau damping which is enhanced as a result of additional tune spread produced by the space charge. The only mode which is never prone to this kind of stabilization is the so-called rigid one.

However, the results of Ref. [4] were obtained with the assumption that space charge tune shift is small in comparison to synchrotron frequency - the condition which is not commonly available. Later the conclusions were confirmed without similar restrictions by numerical investigation of some comparatively simple models (boxcar, square potential well) [5]. Low synchrotron frequency limit was investigated recently in Ref. [6] at arbitrary distribution.

In presented work, the problem is considered at arbitrary distribution function, both at small and large relation of tune shift to synchrotron frequency. The main assumption is that the expected instability growth rate is small enough to satisfy the inequalities:

$$
\operatorname{Im} \omega \ll \Omega_{s}, \quad \operatorname{Im} \omega \ll \Omega_{0} \Delta Q,
$$

where $\Omega_{s}$ and $\Omega_{0}$ are synchrotron and revolution frequencies, and $\Delta Q$ is space charge tune shift. The assumption allows one to solve the problem in two stages: (i) determination of the bunch eigenmodes with space charge only, (ii) investigation of their instability produced

\footnotetext{
*balbekov@fnal.gov
}

by real additions to the impedance. The first point is considered in detail in this article including the following items: (i) The integral-differential equation of the eigenmodes is derived. (ii) Its exact analytical solution is presented for the boxcar bunch to be applied further for testing of different particular models. (iii) An approximate differential equation is developed in the low synchrotron frequency limit, and its solution is presented for different distribution functions. (iv) The same distributions are investigated in the opposite limit of high synchrotron frequency using an uncoupled multipoles model. (v) The applicability of different models is discussed by comparison of these ultimate cases.

It is important that Landau damping manifests itself specifically in this stage as a singularity of corresponding eigenfunctions, so that the dominant space charge almost predetermines the instability threshold. A small real part of the impedance initiates the instability and determines its growth rate, but has almost no affect on the threshold. The formal reason and physical nature of this effect will be discussed below in detail. However, a resemblance of this situation to a coasting beam is seen right now. Indeed, it is well known that the instability threshold of a coasting beam depends, first of all, on a ratio of space charge tune shift to the tune spread. The real addition to the impedance determines the instability growth rate but has almost no affect on the threshold. A peculiarity of bunched beams is that the space charge brings not only tune shift but tune spread as well. Their relation depends on the bunch shape, and investigation of that is an important topic of this paper.

Linear betatron and synchrotron oscillations are considered in the majority of the paper. There are strong grounds to believe that their nonlinearity would enhance Landau damping.

\section{BASIC EQUATION FOR EIGENMODES}

We will start from the equation of betatron oscillations of a single particle: 


$$
\frac{d^{2} x}{d t^{2}}+\Omega^{2} Q^{2} x=\frac{e E[\theta, x-\bar{X}(t, \theta), y]}{m \gamma^{3}}+\frac{e G(t, \theta)}{m \gamma},
$$

where $\Omega$ and $Q$ are momentum-dependent angular velocity and tune, $E$ is space charge electric field, and $e G$ is Lorenz force per particle due to all other sources (wakefield of resistive walls, cavities, etc.). In the beginning, we will neglect this part to find eigenfunctions (e.f.) and eigenvalues (e.v.) of the problem at $G \ll E / \gamma^{2}$. It should be taken into account later as a small perturbation to get additions to the e.v. including the instability growth rate, if it appears.

The field $E$ depends on particle deviations from the bunch center: longitudinal $\theta$ and transverse $x-\bar{X}(t, \theta)$ where $\bar{X}(t, \theta)$ is the beam offset at azimuth $\theta$ ( $y$ offset is presumed to be 0 ). Averaging Eq. (1) over all particles near point $(\theta, u)$ of longitudinal phase space, one can get an equation for the function $X(t, \theta, u)$ which is a local displacement of the beam in this point:

$$
\begin{aligned}
\frac{d^{2} X}{d t^{2}}+\Omega^{2} Q^{2} X= & \frac{e}{m \gamma^{3}} \int_{-\infty}^{\infty} E(\theta, x+X-\bar{X}, y) \\
& \times \rho_{\perp}(x, y) d x d y,
\end{aligned}
$$

where $\rho_{\perp}(x, y)$ is the normalized steady-state beam density. Because both $X$ and $\bar{X}$ are presumed to be small in comparison with the beam diameter, it is possible to expand $E$ in Taylor series obtaining

$$
\frac{d^{2} X}{d t^{2}}+\Omega^{2} Q^{2} X=2 \Omega^{2} Q \Delta Q(\theta)(X-\bar{X}),
$$

where $\Delta Q(\theta)$ is space charge driven tune shift at azimuth $\theta$ averaged over transverse coordinates:

$$
\Delta Q(\theta)=\frac{e}{2 m \gamma^{3} \Omega^{2} Q} \int_{-\infty}^{\infty} \frac{\partial E}{\partial x}(\theta, x, y) \rho_{\perp}(x, y) d x d y
$$

(it is taken into account that $\rho_{\perp}$ and $E$ are even and odd functions of $x$ ). For the elliptical beam of constant density, $\Delta Q$ coincides with the usual incoherent tune shift. For Gaussian beam, $\Delta Q$ is one-half of the tune shift of small betatron oscillations. Other relevant details can be found in Ref. [7].

Because $\Delta Q \ll Q$, Eq. (3) can be reduced to the first order equation:

$$
\frac{d X}{d t}+i \Omega Q X \simeq i \Omega \Delta Q(X-\bar{X}) .
$$

Also, it is necessary to take into account that $d / d t$ is actually a total time derivative including longitudinal motion:

$$
\frac{d}{d t}=\frac{\partial}{\partial t}+\frac{d \theta}{d t} \frac{\partial}{\partial \theta}+\frac{d u}{d t} \frac{\partial}{\partial u}=\frac{\partial}{\partial t}+\Omega_{s} \frac{\partial}{\partial \phi},
$$

where $\phi$ and $\Omega_{s}$ are phase and frequency of synchrotron oscillations, $u \propto p-p_{0}$ is normalized momentum deviation, and subindex " 0 " marks the central momentum.
We will look for solutions of the obtained equation in the form

$$
X(t, \theta, u)=Y(\theta, u)(-i \omega t-i \chi \theta),
$$

where $\chi=(\Omega Q)^{\prime} / \Omega^{\prime}$ is treated as an effective chromaticity, and prime means derivative on momentum. It allows one to exclude dependence of $\Omega$ and $Q$ on momentum in (5) resulting in the equation:

$$
\left(\omega-\Omega_{0} Q_{0}\right) Y+i \Omega_{s} \frac{\partial Y}{\partial \phi}=-\Omega_{0} \Delta Q(\theta)(Y-\bar{Y}) .
$$

Further we will use the notations,

$$
\frac{\omega-\Omega_{0} Q_{0}}{\Omega_{0} \Delta Q(0)}=\nu, \quad \frac{\Omega_{s}}{\Omega_{0} \Delta Q(0)}=\mu,
$$

to represent Eq. (8) in the form

$$
\nu Y+i \mu \frac{\partial Y}{\partial \phi}=-\frac{\rho(\theta)}{\rho(0)}(Y-\bar{Y}),
$$

where $\rho(\theta)$ is linear bunch density at longitudinal distribution function $F$ :

$$
\rho(\theta)=\int_{-\infty}^{\infty} F(\theta, u) d u .
$$

Equation (10) should be supplemented by a relation of variables $Y$ and $\bar{Y}$ :

$$
\rho(\theta) \bar{Y}(\theta)=\int_{-\infty}^{\infty} F(\theta, u) Y(\theta, u) d u
$$

which is valid for the pair $X-\bar{X}$ as well.

In fact, Eq. (10) has been derived first in my work [4] based on the Vlasov equation for the function $D=F Y$ which is linear density of the beam dipole moment. The conversion is trivial, but form (10) is used in this paper as more convenient.

Parameter $\nu$ can be treated as an eigenvalue of Eq. (10). It is easy to show that all of the e.v. are real numbers, and corresponding e.f. satisfy the orthogonality condition:

$$
\iint Y_{i} Y_{j}^{*} F d \theta d u=\delta_{i j}, \quad \operatorname{Im} \nu=0 .
$$

Note one important exact solution which is valid at any distribution function:

$$
Y=\bar{Y}=\text { const, } \quad \nu=0, \quad \text { i.e. } \omega=\Omega_{0} Q_{0} .
$$

We will treat it further as a "rigid mode" because it does not depend on synchrotron oscillations (this term was primarily used for a model without synchrotron motion at all; for example, see Ref. [8]). Because of orthogonality condition (13), all other e.f. should satisfy the relations

$$
\iint Y_{i} F d \theta d u=\int \bar{Y}_{i}(\theta) \rho(\theta) d \theta=\int \bar{D}_{i}(\theta) d \theta=0,
$$

which means that the total dipole moment of the bunch is 
zero (at zero effective chromaticity). It is easy to believe that wakefield of similar modes and attendant instability (if it appears) would be noticeably lower than with the rigid mode.

Further we will consider linear synchrotron oscillations taking the bunch half length as 1 :

$$
\theta=A \cos \phi, \quad u=A \sin \phi, \quad A \leq 1,
$$

where $A=\sqrt{\theta^{2}+u^{2}}$ is the amplitude of synchrotron oscillations. Their nonlinearity is not included here because Landau damping from space charge is the main topic of the paper.

In conclusion of this section, it is necessary to note that the substitution $X \propto \exp (-i \omega t)$ means, strictly saying, Laplace transformation of Eq. (5), an operation which is determined at $\operatorname{Im} \omega>0$. Generally, the transformed equation should include initial function $X(0, \theta, u)$ as well. It is traditionally omitted because the instability threshold and growth rate do not depend on this. However, it is important to emphasize that the complete equation has a solution at any complex $\nu$ with a positive imaginary part. Real $\nu$, including e.v. of uniform Eq. (10), have a direct meaning only if the corresponding e.f. are a regular function defined as the analytical extension of the original complex function on the real axis. Otherwise, it would be required to bypass the pole at inverse Laplace transformation, an operation which specifically provides Landau damping [9].

Therefore, only regular solutions of Eq. (10) have a chance to become unstable due to the addition $\propto G$ in Eq. (1) (for example, rigid mode). Other (singular) modes are prone to Landau damping, and only strong perturbation $G$ could cause their instability by the essential transformation of the original spectrum. However, similar cases are not a subject of this exploration. For briefness, below we will identify mentioned modes simply as "unstable" or "stable" ones.

\section{BOXCAR MODEL: EXACT SOLUTION}

The distribution function providing constant linear density (boxcar model) is

$$
F=\frac{1}{\pi \sqrt{1-\theta^{2}-u^{2}}}, \quad \rho=1 \quad \text { at }|\theta|<1 .
$$

The first exact solution of the problem in the frame of this model is presented by Sacherer [3] in the form of Legendre polynomials:

$$
\bar{Y}(\theta) \propto P_{n}(\theta), \quad n=0,1,2, \ldots
$$

Substitution to Eq. (10) shows that, at any $n$, there are $n+$ 1 different e.v. $\nu$ and corresponding e.f. $Y(\theta, u)$ which are polynomials of the same power as well. It provides an excellent opportunity to check any subsequent partial model. Some examples given below will be used in the next sections:

$$
\begin{array}{ll}
\mathbf{n}=\mathbf{0} & \bar{Y}=Y=1, \quad \nu=0, \quad \text { (rigid mode); } \\
\mathbf{n}=\mathbf{1} & \bar{Y}=\theta, \quad Y=\theta+\frac{i \nu u}{\mu}, \quad \nu=-0.5 \pm \sqrt{\mu^{2}+0.25} \\
\mathbf{n}=\mathbf{2} & \bar{Y}=3 \theta^{2}-1, \quad \hat{\nu}\left(\hat{\nu}^{2}-4 \mu^{2}\right)=\hat{\nu}^{2}-\mu^{2}, \quad \hat{\nu}=\nu+1 ; \\
\mathbf{n}=\mathbf{3} & \bar{Y}=5 \theta^{3}-3 \theta, \quad\left(\hat{\nu}^{2}-\mu^{2}\right)\left(\hat{\nu}^{2}-9 \mu^{2}\right)=\hat{\nu}\left(\hat{\nu}^{2}-4 \mu^{2}\right)
\end{array}
$$

(a continuation of the table is quite apparent). The eigenvalues $\nu$ are plotted in Fig. 1 vs $\mu$ at $n=0-5$. It is seen that, at large $\mu$, the eigenmodes form several groups with close frequencies and about circular polarization:

$$
\begin{aligned}
& Y_{m, n} \simeq R_{m, n}(A) \exp (i m \phi), \quad \nu \simeq m \mu, \\
& \omega \simeq \Omega_{0} Q_{0}+m \Omega_{s}
\end{aligned}
$$

which coincide with "classic" head-tail modes [1-3]. It is seen also that the radial modes $R_{m, n}(A)$ arise here from different Legendre polynomials $P_{n}(\theta)$.

At lower $\mu$, any e.v. tends to one of two points: (i) either $\nu=0$, (ii) or $\nu=-1$. In standard terms, frequencies of these modes are

$$
\omega \simeq \Omega_{0} Q_{0} \quad \text { or } \quad \Omega \simeq \Omega_{0}\left(Q_{0}-\Delta Q\right)=\Omega_{0} Q_{\text {incoherent }} .
$$

These oscillations have almost linear polarization: along $\theta$ in the first case, and along $u$ in the second one. Because of the frequencies coalescing, there is almost total degeneration of corresponding e.f. at very small $\mu$. In particular, two groups of solutions can be formally obtained directly from Eq. (10) at $\mu=0$. The first of them includes arbitrary e.f. $Y(\theta)$ not depending on $u$. According to Eq. (12), $\bar{Y}=Y$ in this case, and Eq. (10) is satisfied at any $\rho(\theta)$, if $\nu=0$. The other group includes arbitrary e.f. $Y(\theta, u)$ satisfying the condition $\int F Y d u=0$ (for example, any odd function of $u$ ). Then $\bar{Y}=0$, and Eq. (10) is satisfied at $\rho(\theta)=$ const, if $\nu=-1$.

Of course, these sets cannot provide a basis for further development. However, at very realistic conditions $\operatorname{Im} \omega \ll \Omega_{s}$, synchrotron motion could remove the degeneration and form the modes available for subsequent generalization.

Therefore, our first goal is to use these ideas for analysis of more realistic distributions at $\mu \ll 1$. The case $\nu \simeq 0$ is rather clear in this regard, offering an expansion of the 


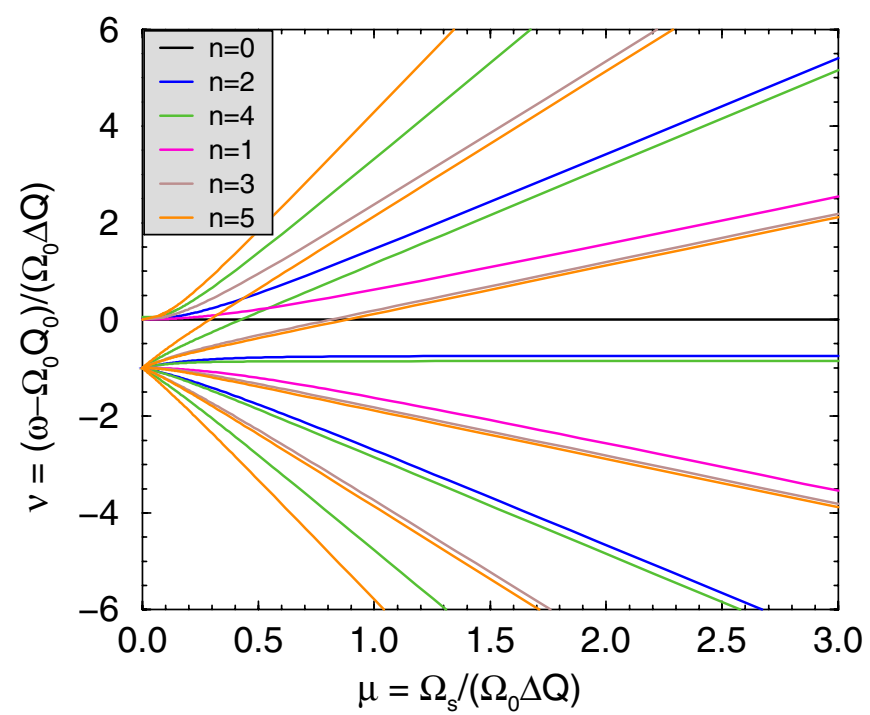

FIG. 1. (Color) Exact eigenvalues of the boxcar bunch.

solutions in powers of $u$. The other possibility $\nu \simeq-1$ is more complicated because of the crucial condition $\rho(\theta)=$ const which ensures constant incoherent frequency of all the particles. Then different parts of the bunch can oscillate jointly with the same coherent frequency creating various spatial configurations with $\bar{Y}=0$. However, only a short part of the bunch could oscillate with a definite frequency if $\rho(\theta) \neq$ const. Then the term $\mu \partial Y / \partial \phi$ could not be treated as a small perturbation as it looks like $0 \times \infty$.

However, instability of similar oscillations seems unlikely because: (i) Landau damping affects when coherent frequency is located inside incoherent distribution, (ii) actually, relation $\bar{Y} \simeq 0$ means a low wakefield. Therefore, only the first case is considered in the next section.

\section{LOW SYNCHROTRON FREQUENCY APPROACH}

We will look for the solution of Eq. (10) at $\mu \ll 1$ in the form

$$
Y=y_{0}(\theta)+y_{1}(\theta) u+y_{2}(\theta) u^{2}, \quad\left|y_{0}\right| \gg\left|y_{1}\right| \gg\left|y_{2}\right| .
$$

Then, according to Eq. (12)

$$
\bar{Y}(\theta)=y_{0}(\theta)+y_{2}(\theta) U^{2}(\theta),
$$

where $U(\theta)$ is normalized rms momentum:

$$
U^{2}(\theta)=\frac{\int F(\theta, u) u^{2} d u}{\int F(\theta, u) d u}
$$

Substituting it in Eq. (10) and neglecting the terms $\propto \mu y_{2}$, we obtain

$$
\begin{aligned}
& \nu\left(y_{0}+y_{1} u+y_{2} u^{2}\right)+i \mu\left(-y_{0}^{\prime} u-y_{1}^{\prime} u^{2}+y_{1} \theta\right) \\
& \quad \simeq-\frac{\rho}{\rho(0)}\left[y_{1} u+y_{2}\left(u^{2}-U^{2}\right)\right],
\end{aligned}
$$

where prime means derivative on $\theta$. It gives the relations

$$
\begin{aligned}
& y_{1}=\frac{i \mu y_{0}^{\prime}}{\nu+\rho / \rho(0)}, \\
& y_{2}=-\frac{\mu^{2}}{\nu+\rho / \rho(0)}\left(\frac{y_{0}^{\prime}}{\nu+\rho / \rho(0)}\right)^{\prime},
\end{aligned}
$$

and the differential equation for $y_{0}$ :

$$
\nu y_{0}=\frac{\mu^{2}}{\nu+\rho / \rho(0)}\left[y_{0}^{\prime} \theta-\frac{\rho U^{2}}{\rho(0)}\left(\frac{y_{0}^{\prime}}{\nu+\rho / \rho(0)}\right)^{\prime}\right] .
$$

Using Eqs. (20) and (22), one can represent $y_{0}$ in terms of $\bar{Y}$. With the same accuracy as before, the relation is

$$
y_{0} \simeq \bar{Y}+\frac{\mu^{2} U^{2}}{\nu+\rho / \rho(0)}\left(\frac{\bar{Y}^{\prime}}{\nu+\rho / \rho(0)}\right)^{\prime} .
$$

Finally, it gives the following equation for the function $\bar{Y}(\theta)$ :

$$
U^{2} \bar{Y}^{\prime \prime}-\left(\theta+\frac{U^{2} \rho^{\prime} / \rho(0)}{\nu+\rho / \rho(0)}\right) \bar{Y}^{\prime}+\frac{\nu[\nu+\rho / \rho(0)]}{\mu^{2}} \bar{Y}=0 .
$$

Because the equation has solutions at any $\nu$, additional conditions are required to select the satisfying basic Eq. (10). First of all, it is the rigid mode $\nu=0, \bar{Y}=1$. All other modes should satisfy orthogonality condition (15) which is sufficient to sort all even functions $\bar{Y}(\theta)$. For odd ones, multiply Eq. (10) by $F \theta$ and integrate it over $d \theta d u$. Then, applying Eqs. (19), (23), and (25) with accepted accuracy, one can get the required condition:

$$
\int \bar{Y}(\theta) \rho(\theta) \theta d \theta \simeq \frac{\mu^{2}}{\nu} \int \frac{U^{2}(\theta) \bar{Y}^{\prime}(\theta) \rho(\theta) d \theta}{\nu+\rho(\theta) / \rho(0)} .
$$

\section{A. Boxcar model}

Boxcar bunch model (17) is considered in this subsection again. Their characteristic functions are

$$
\rho(\theta)=1, \quad U^{2}(\theta)=\frac{1-\theta^{2}}{2} \quad \text { at }|\theta|<1 .
$$

Therefore Eq. (26) transforms to the Legendre equation:

$$
\left(1-\theta^{2}\right) \bar{Y}^{\prime \prime}-2 \theta \bar{Y}^{\prime}+\frac{2 \nu(\nu+1)}{\mu^{2}} \bar{Y}=0 .
$$

Legendre polynomials (18) are the solutions satisfying conditions (15) and (27), and the dispersion equation for corresponding e.v. is

$$
\frac{2 \nu(\nu+1)}{\mu^{2}}=n(n+1), \quad \text { at } \bar{Y}(\theta)=P_{n}(\theta) .
$$




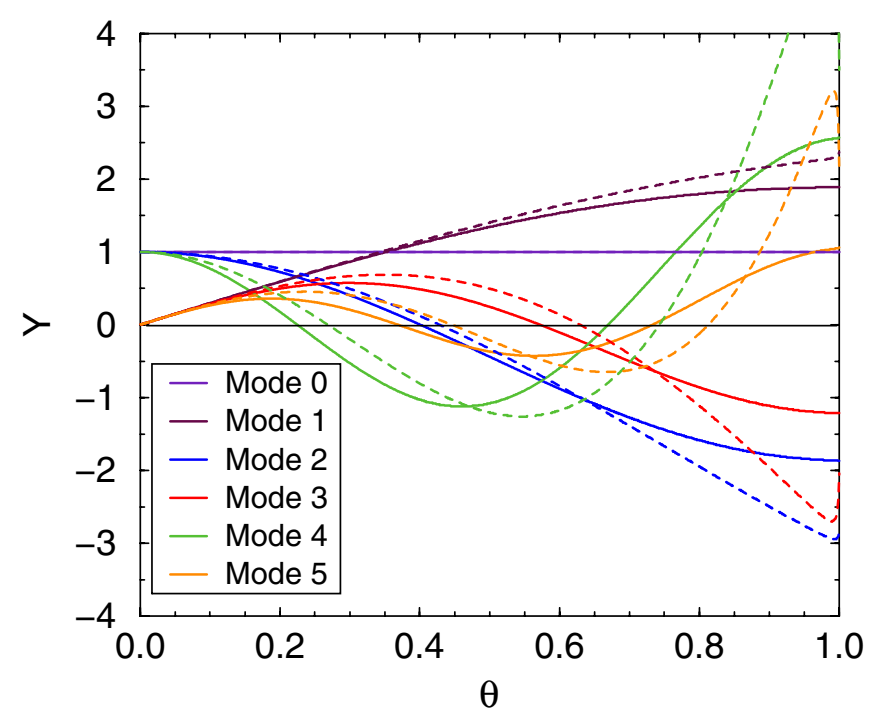

FIG. 2. (Color) Eigenfunctions of the parabolic bunch vs azimuth. Solid lines: $\mu=0.05$; dashed ones: $\mu=1$.

Taking into account the basic assumptions of this section, we must use only the eigenvalues:

$$
\nu=\frac{1}{2}\left[\sqrt{1+2 \mu^{2} n(n+1)}-1\right] \simeq \frac{\mu^{2} n(n+1)}{2} .
$$

Note that they satisfy the equation of Sec. III with a precision at least of $\mu^{2}$ at any $n$, and exactly at $n=1$.

\section{B. Parabolic and "superparabolic" models}

The more realistic parabolic bunch has the characteristics

$$
F=\frac{2}{\pi} \sqrt{1-A^{2}}, \quad \rho=1-\theta^{2}, \quad U^{2}=\frac{1-\theta^{2}}{4} .
$$

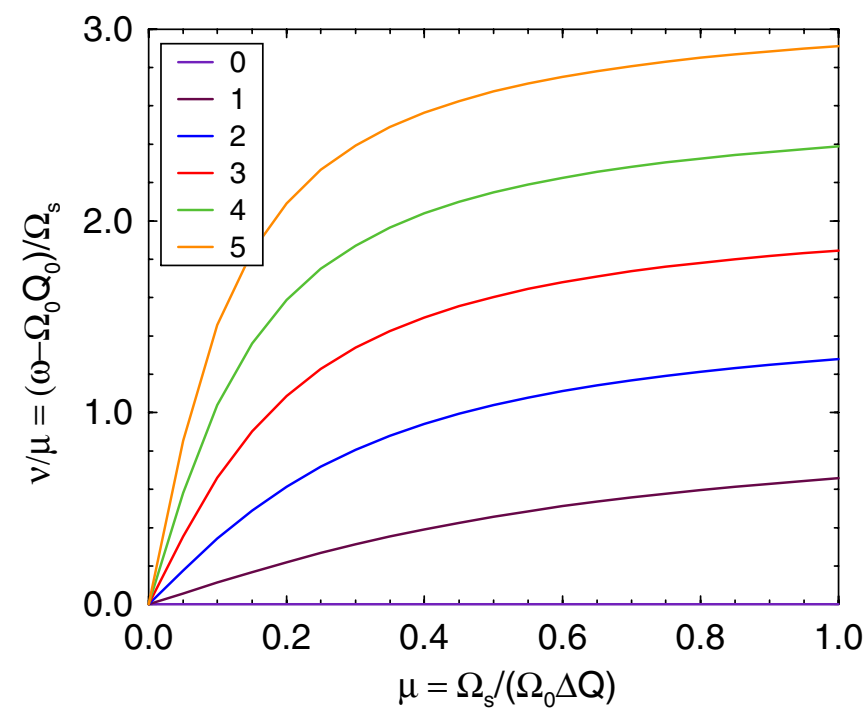

FIG. 3. (Color) Normalized eigenvalues of the parabolic bunch.

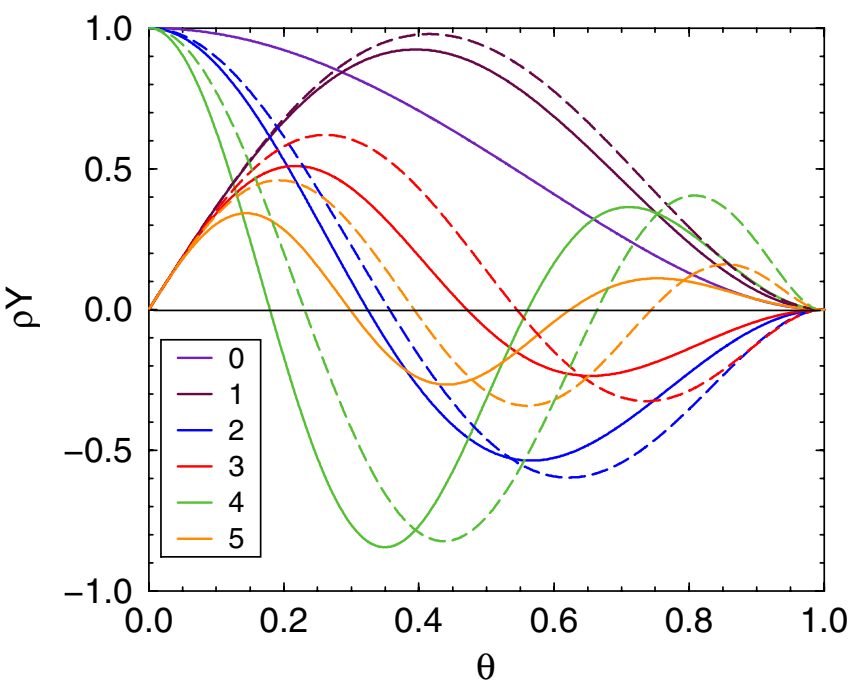

FIG. 4. (Color) Eigenfunctions of the "superparabolic" bunch. Solid lines: $\mu=0.05$; dashed ones: $\mu=1$.

Some of its e.f. and corresponding e.v. are plotted in Figs. 2 and 3 (the range $0 \leq \theta \leq 1$ is shown here and further because all the e.f. are even or odd functions of $\theta$ ). It is seen that these e.v. do not significantly differ from the boxcar ones as it is described by Eq. (31). However, it is important to emphasize that e.f. depend on parameter $\mu$ now, and there is a growth of these functions at the bunch tails if the parameter is greater.

Distribution function (32) has an infinite derivative on the boundary. This circumstance could qualitatively effect on the bunch behavior. Therefore, we consider a smoother "superparabolic" distribution:

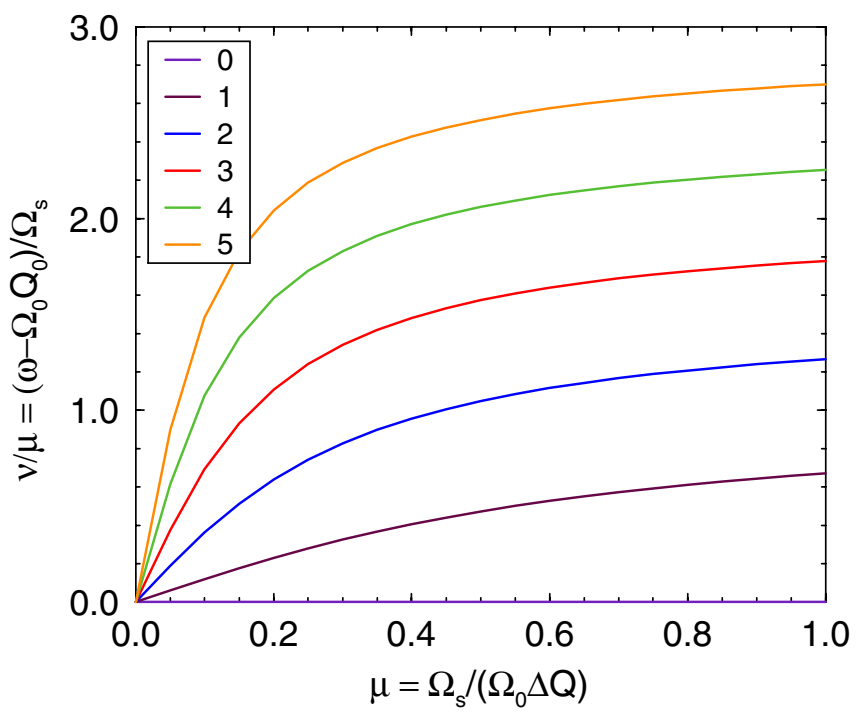

FIG. 5. (Color) Normalized eigenvalues of the "superparabolic" bunch. 


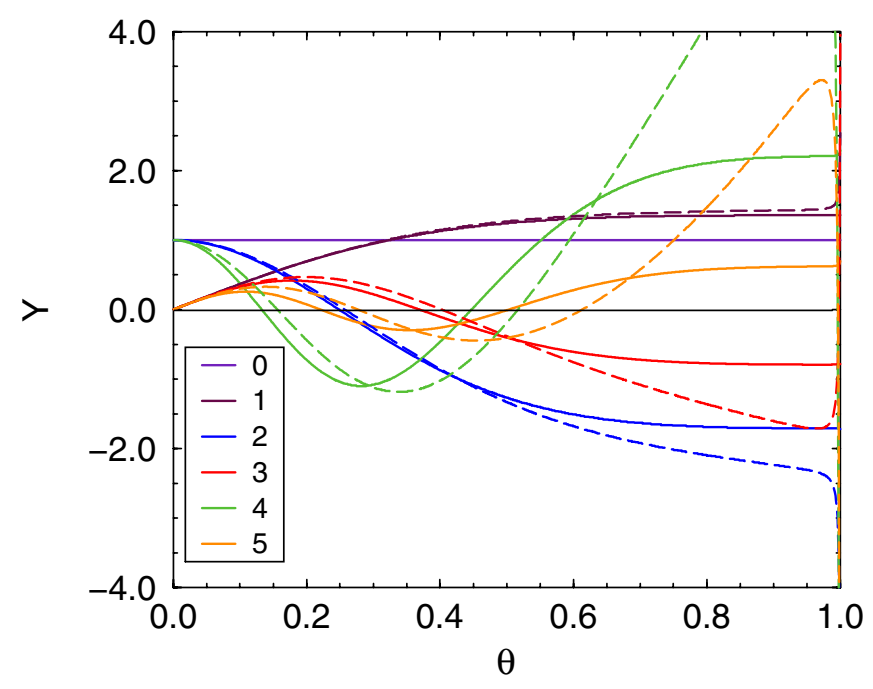

FIG. 6. (Color) Eigenfunctions of the Gaussian bunch at $\sigma=$ $1 / 3$ and low $\mu$. Solid lines: $\mu=0.01$; dashed ones: $\mu=0.2$.

$$
\begin{gathered}
F=\frac{8}{3 \pi}\left(1-A^{2}\right)^{3 / 2}, \quad \rho=\left(1-\theta^{2}\right)^{2}, \\
U^{2}=\frac{1-\theta^{2}}{6} .
\end{gathered}
$$

The results are presented in Figs. 4 and 5. In this case, all of the e.f. $\bar{Y}(\theta)$ other than the rigid mode demonstrate very fast growth in the bunch tails, especially at large $\mu$. Therefore the functions $D=\rho \bar{Y}$ are plotted in Fig. 4 instead of $\bar{Y}(\theta)$ (including the rigid mode).

\section{Gaussian bunch}

Because Gaussian distribution is always truncated in reality, we will consider the distribution function which

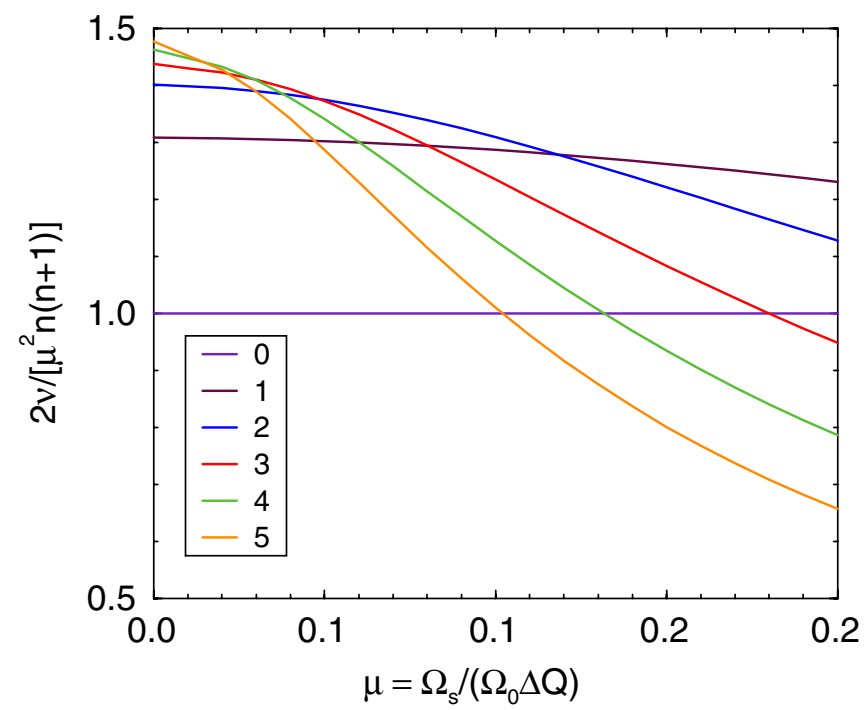

FIG. 7. (Color) Eigenvalues of the Gaussian bunch at $\sigma=1 / 3$, low $\mu$.

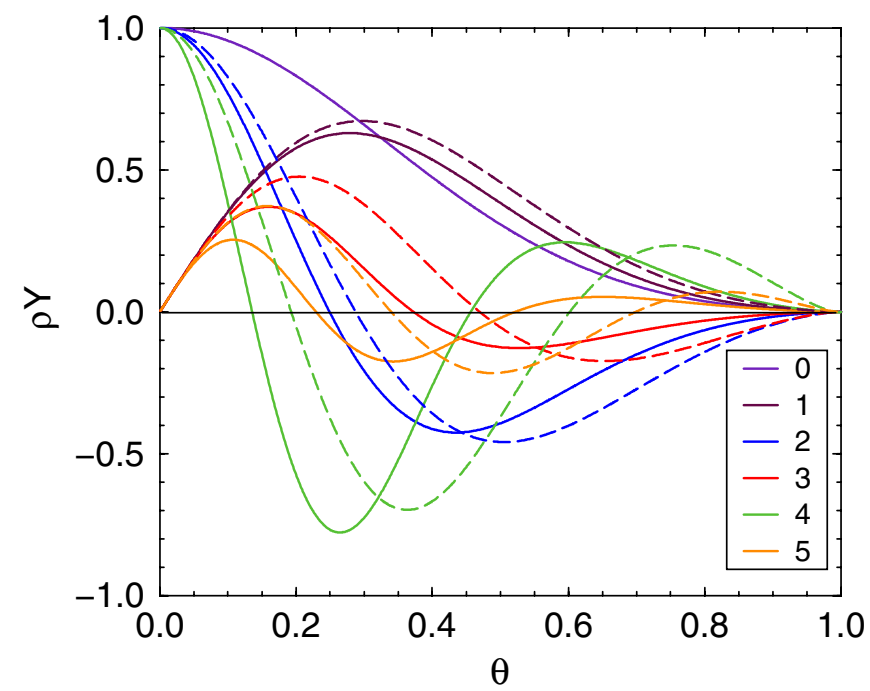

FIG. 8. (Color) Eigenfunctions of the Gaussian bunch at higher $\mu$. Solid lines: $\mu=0.05$; dashed ones: $\mu=1$.

provides a bounded bunch with smooth tails:

$$
F \propto \exp \left(\frac{1-A^{2}}{2 \sigma^{2}}\right)-1 \quad \text { at } A<1
$$

Other characteristics of the bunch are

$$
\rho(\theta)=\sigma \sqrt{\frac{\pi}{2}} \exp \left(\frac{1-\theta^{2}}{2 \sigma^{2}}\right) \operatorname{erf}\left(\frac{\sqrt{1-\theta^{2}}}{\sigma \sqrt{2}}\right)-\sqrt{1-\theta^{2}},
$$

and

$$
U^{2}(\theta)=\sigma^{2}-\frac{\left(1-\theta^{2}\right)^{3 / 2}}{3 \rho(\theta)} .
$$

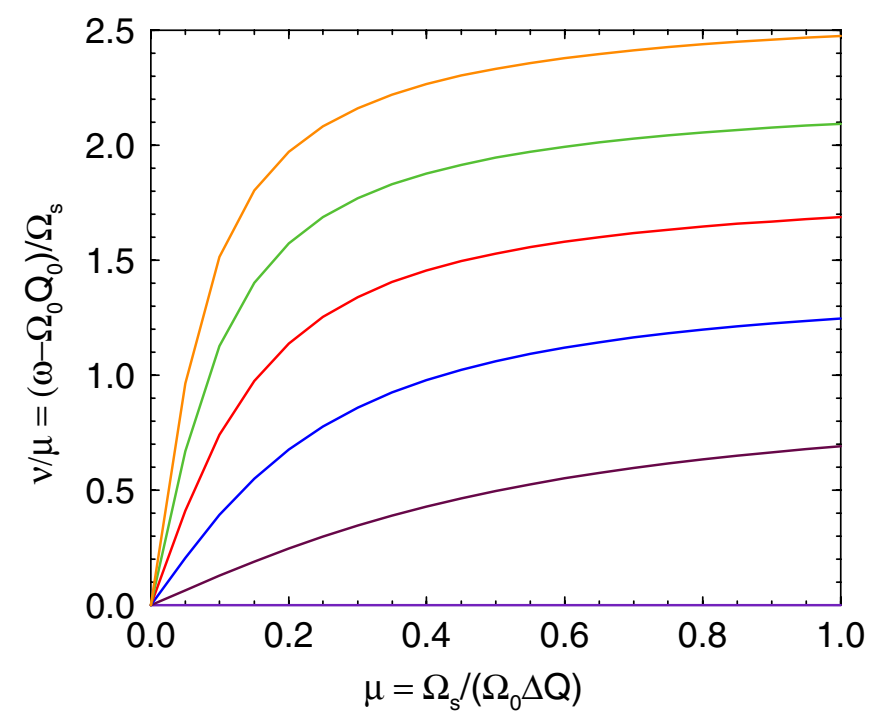

FIG. 9. (Color) Eigenvalues of the Gaussian bunch at $\sigma=1 / 3$, higher $\mu$. 
Results of calculations are presented in Figs. 6-9 at $\sigma=$ $1 / 3$. It is seen that all of the eigenfunctions tend to constant on the bunch tails at $\mu=0.01$. However, they grow rapidly at $\mu=0.2$, and very rapidly at larger $\mu$. To mitigate the plots, density of the dipole moment $D=\rho \bar{Y}$ is presented in Fig. 8 instead of $\bar{Y}$.

\section{Low $\mu$ summary}

It is shown in this section that, at $\mu \lesssim 0.1$, all observed e.f. are regular functions of their arguments, and e.v. are not too different from those of the boxcar model. However, transverse deviation heavily increases in the bunch tails at larger $\mu$, and the possibility must not be ruled out that a singularity appears at $\mu \sim 1$ (numerical accuracy does not allow one to make such a definite conclusion, because observed growth of the functions is always restricted in the calculations). As it is indicated in the end of Sec. II, similar behavior could be a sign of Landau damping.

However, it is necessary to remember that the assumption $\mu \ll 1$ lies in the heart of this section, so that the extrapolation to $\mu \sim 1$ is not quite legitimate and must be treated as a hypothesis only. Therefore, for additional confirmation, the opposite limit $\mu \gg 1$ is explored in the following section. Though this instance was investigated in my earlier paper [4], additional information is presented here with a final goal to develop a more complete pictureat least qualitatively.

\section{HIGH SYNCHROTRON FREQUENCY APPROACH}

Because beam deviation $Y$ is a periodical function of synchrotron phase, it can be presented in the form

$$
Y=\sum_{n} Y_{n}(A) \exp (i n \phi) .
$$

Let us substitute it in Eq. (10), multiply the equation by $\exp (-i m \phi)$, and integrate over $\phi$ using relations (16). It results in a series of coupled integral equations:

$$
\begin{aligned}
(\nu-m \mu) Y_{m}(A)= & -\sum_{m^{\prime}} R_{m-m^{\prime}}(A) Y_{m^{\prime}}(A) \\
& +\sum_{m^{\prime}} \int_{0}^{1} K_{m, m^{\prime}}\left(A, A^{\prime}\right) Y_{m^{\prime}}\left(A^{\prime}\right) d A^{\prime}
\end{aligned}
$$

with coefficients

$$
\begin{gathered}
R_{m-m^{\prime}}(A)=\frac{1}{\pi \rho_{0}} \int_{-A}^{A} \frac{T_{m-m^{\prime}}(\theta / A)}{\sqrt{A^{2}-\theta^{2}}} \rho(\theta) d \theta, \\
K_{m, m^{\prime}}\left(A, A^{\prime}\right)=\frac{2 F\left(A^{\prime}\right) A^{\prime}}{\pi \rho_{0}} \int_{-a}^{a} \frac{T_{m}(\theta / A)}{\sqrt{A^{2}-\theta^{2}}} \frac{T_{m^{\prime}}\left(\theta / A^{\prime}\right)}{\sqrt{A^{\prime 2}-\theta^{2}}} d \theta,
\end{gathered}
$$

where $T_{m}(x)=\cos (m \arccos x)$ are Chebyshev polynomials, and $a=\min \left(A, A^{\prime}\right)$. If $\mu \gg 1$, coupling of the multipoles can be neglected, and the series is reduced to a set of independent equations:

$$
\begin{aligned}
\Lambda_{m, j} Y_{m, j}(A)= & \int_{0}^{1}\left[K_{m, m}\left(A, A^{\prime}\right)-R_{0}(A) \delta\left(A^{\prime}-A\right)\right] \\
& \times Y_{m, j}\left(A^{\prime}\right) d A^{\prime},
\end{aligned}
$$

where $\Lambda_{m, j}=\nu-m \mu$ are treated as eigenvalues, and $j=$ $1,2, \ldots$ are the eigenmode numbers. Solutions of these equations are known as radial modes [3]. One can see again that the rigid mode $Y_{0,1}(A)=1, \Lambda_{0,1}=0$ is an exact solution of Eq. (41) independently on the distribution function. Other modes will be examined numerically using the following method.

Replacement $Z=Y \sqrt{F A}$ and shift $\Lambda^{\prime}=\Lambda+2$ are applied to get an equation with symmetric positive definite kernel. The next step is a change of the integral by a sum to get a series of linear equations with symmetric square matrix (typically $100 \times 100$, sometimes $500 \times 500$ ). The fast iteration method is applied to find maximal e.v. $\Lambda^{\prime}$ and corresponding e.f. After the matrix reduction, the process has to be repeated for the next e.v., etc.

\section{A. Boxcar model}

Several e.v. and e.f. of the boxcar bunch (17) are presented in Table I and Fig. 10. They can be found also by using exact solutions of Sec. III. Corresponding values are given in Table I as rational fractions in round brackets, and orders of used Legendre polynomials are placed in square brackets. It is seen that, at any $m$, successive solutions (radial modes) are caused by the polynomials of order $n=$ $|m|,|m|+2$, etc. At $m=0$, radial modes obtained by Fourier expansion of the exact solutions are

$$
\begin{aligned}
& Y_{0,1}=1, \quad Y_{0,2}=1-\frac{3 A^{2}}{2}, \\
& Y_{0,3}=1-5 A^{2}+\frac{35 A^{4}}{8}, \cdots
\end{aligned}
$$

TABLE I. Eigenvalues of the boxcar bunch.

\begin{tabular}{lccc}
\hline \hline$m$ & $j=1$ & $j=2$ & $j=3$ \\
\hline 0 & $-0.01(0)[0]$ & $-0.75(-3 / 4)[2]$ & $-0.86(-55 / 64)[4]$ \\
1 & $-0.51(-1 / 2)[1]$ & $-0.81(-13 / 16)[3]$ & $-0.88(-113 / 128)[5]$ \\
2 & $-0.63(-5 / 8)[2]$ & $-0.84(-27 / 32)[4]$ & $-0.90(-919 / 1024)[6]$ \\
\hline \hline
\end{tabular}




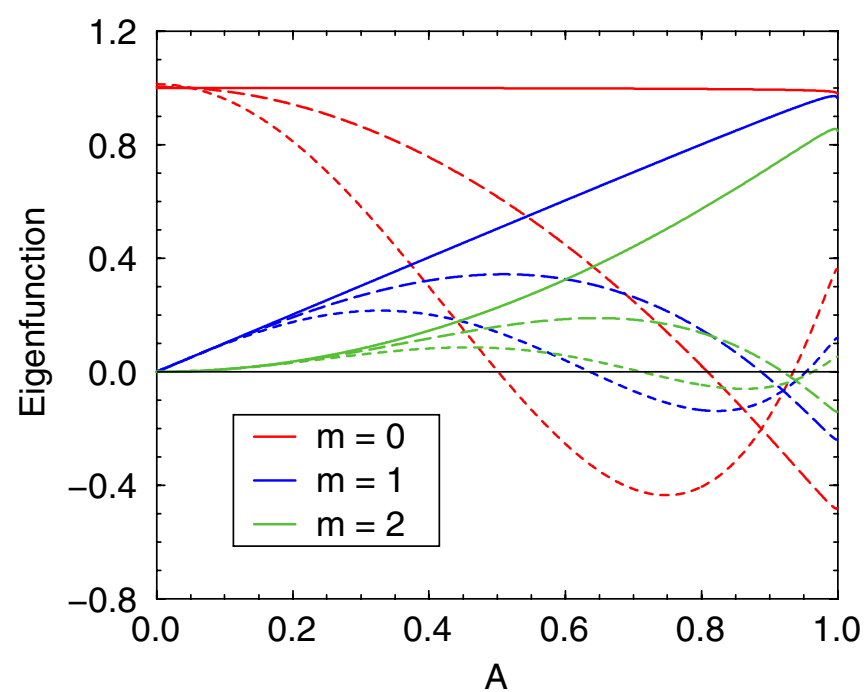

FIG. 10. (Color) Eigenfunctions of the boxcar bunch vs normalized synchrotron amplitude (uncoupled multipoles approach). Three radial modes are presented for each multipole.

being in a good agreement with numerical results presented in Fig. 10. The same is true for higher modes.

\section{B. Parabolic model}

In contrast with the boxcar, the space charge tune shift of the parabolic model (32) depends on particle position, bringing an additional tune spread which is a key point of Landau damping. As mentioned above, the damping should appear as a singularity of corresponding eigenfunctions at the eigenvalues fallen to the incoherent range.

The numerical solution of Eq. (41) has demonstrated that only the lowest radial modes have no singularities at

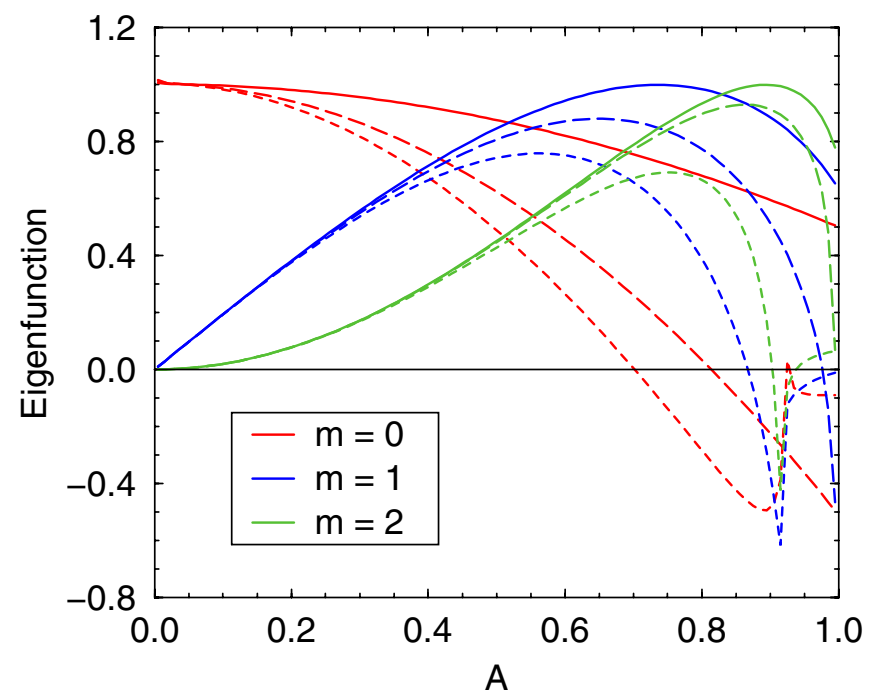

FIG. 11. (Color) Modified e.f. of the parabolic bunch vs normalized synchrotron amplitude (three radial modes for each multipole).

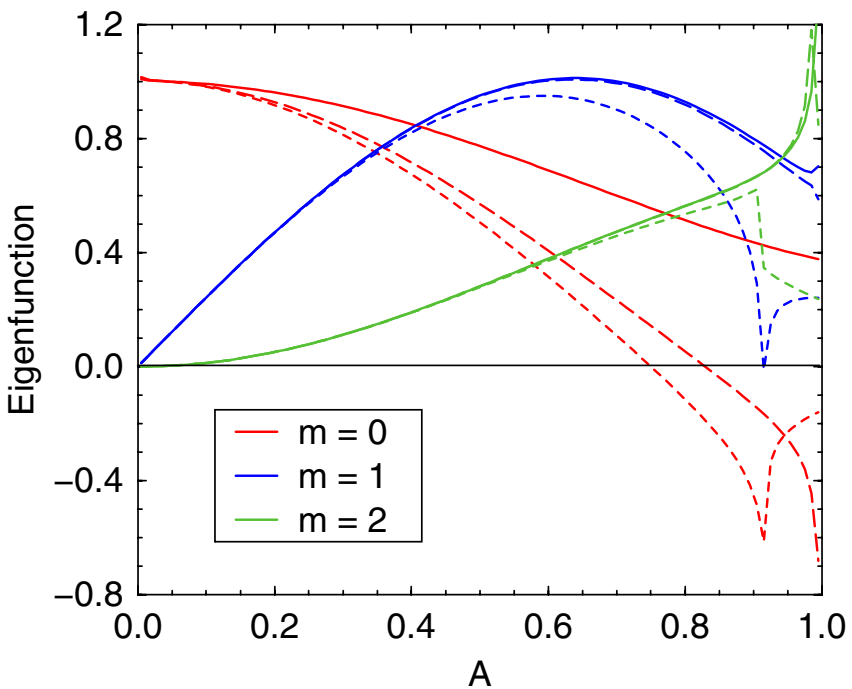

FIG. 12. (Color) Modified eigenvalues of the "superparabolic" bunch.

$m=0,1$, and 2 (other multipoles were not examined). Eigenvalues of these modes are

$$
\Lambda_{0,1}=0, \quad \Lambda_{1,1}=-0.422, \quad \Lambda_{2,1}=-0.482 .
$$

All other investigated modes form a continuous spectrum at $-1<\Lambda_{m, j}<-0.5$. Because all related eigenfunctions are singular, continuous combinations are presented in Fig. 11:

$$
\tilde{Y}_{m, j}(A)=\left[\Lambda_{m, j}+R_{0}(A)\right] Y_{m, j}(A), \quad R_{0}=1-\frac{A^{2}}{2}
$$

including regular (unstable) lowest modes. Note that stable functions which were singular originally have a break of derivative in this format.

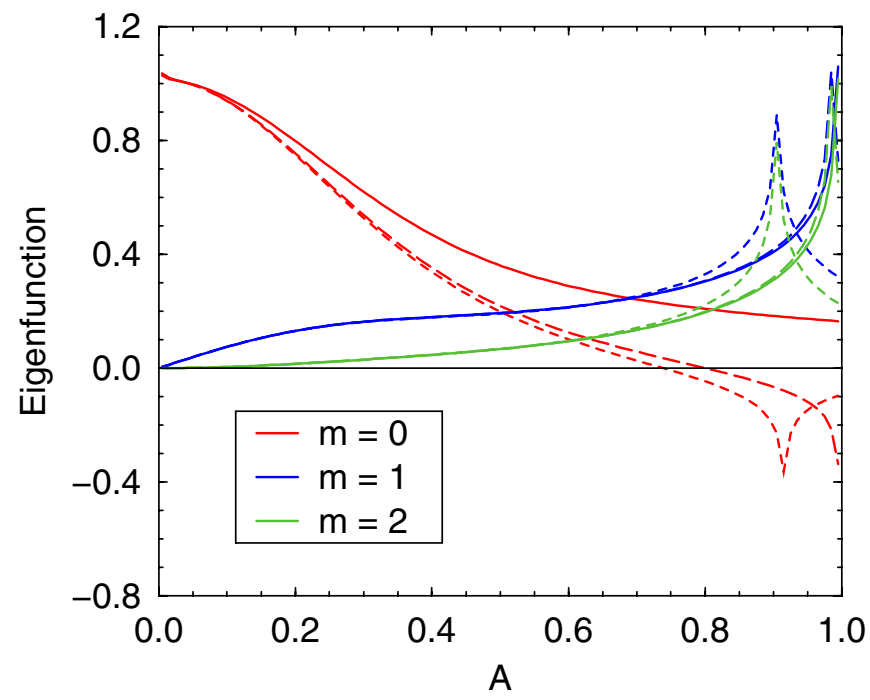

FIG. 13. (Color) Modified e.f. of the Gaussian bunch. 


\section{C. "Superparabolic" and Gaussian bunches}

In contrast with previous examples, the "superparabolic" bunch (33) has no derivative abruption on the boundary. Probably because of this the rigid mode $(0,1)$ is the only regular solution of Eq. (41), whereas all other modes have the singularity and form a continuous spectrum at $-1 \leq \Lambda \leq-3 / 8$. Some of the modes are presented in Fig. 12 in the format like (42).

A very similar picture appears at the Gaussian distribution described by Eqs. (34)-(36). Once again, the rigid mode is the only regular (potentially unstable) solution. Some other e.f. are plotted in Fig. 13 at $\sigma=1 / 3$ when the continuous spectrum is located at $-1 \leq \Lambda \leq-0.274$.

\section{High $\boldsymbol{\mu}$ summary}

Considered examples demonstrate that, at $\mu \gg 1$ and any realistic distribution, the coherent spectrum includes several distinct frequencies and continuum. The distinct eigenmodes are regular functions of their arguments and should be treated as potentially unstable. Any e.f. in the continuum have a singularity and are prone to Landau damping. The number of the distinct (unstable) modes is less at smoother distribution, and the rigid mode is the only one that can be unstable in a long-tail distribution like Gaussian.

A formal interrelation of Landau damping and e.f. singularity has been commented on at the end of Sec. II. Now, it is pertinent to add a physical explanation of the phenomenon.

Two space charge produced effects are important for a bunched beam: (i) shifts of coherent eigenmodes from bare betatron tune, (ii) incoherent tune spread depending on the particle position. Landau damping appears when the second effect dominates, and coherent frequency falls into an incoherent distribution. Then the electromagnetic wave linked to the coherent motion strongly excites aboutresonant particles creating a singularity and suffering energy loss. It is important that the particles are excited not in phase, so that their forced oscillations are incoherent and do not support the coherent motion which is damping in the end.

It is clear that the result depends on the bunch shape. For example, the damping is completely absent in the boxcar bunch because all of its particles have the same tune and are excited in phase, supporting coherent motion. But the damping is very important for realistic long-tail distributions, because their tune spread is rather large to overcome the coherent tune shift. That is the reason why the only rigid mode can be unstable in the Gaussian bunch.

\section{COMPARISON AND COUPLING OF THE MODELS}

The foregoing results were obtained at very extreme assumptions: either $\mu \ll 1$ or $\mu \gg 1$ (below:

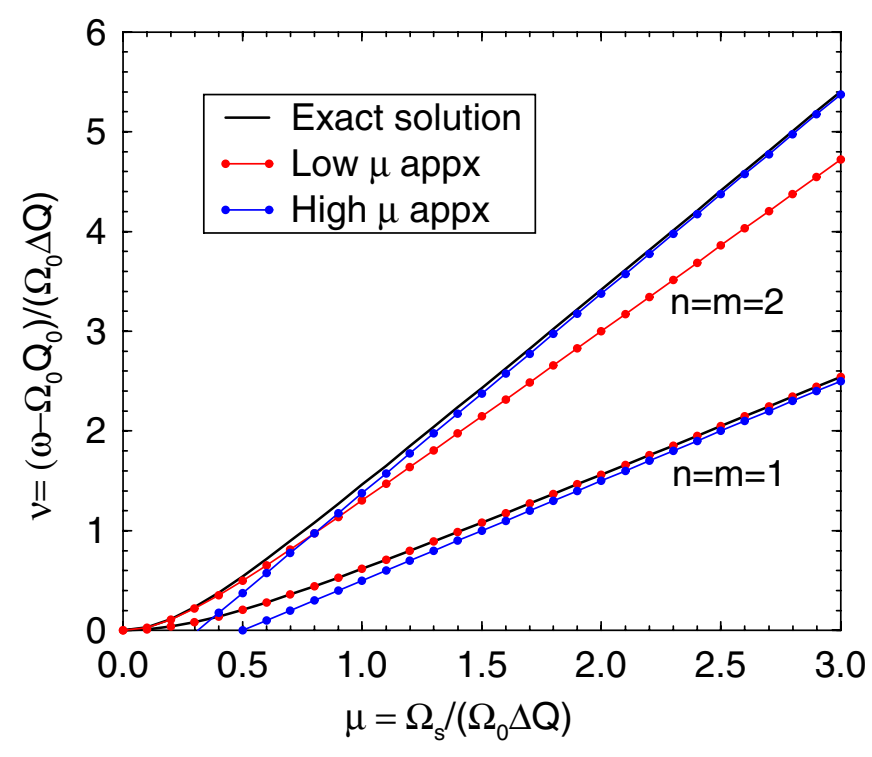

FIG. 14. (Color) Eigenvalues of the boxcar bunch. Low $\mu$ approximation perfectly coincides with an exact solution at $n=1$.

low/high- $\mu$ approximations). Correlation of these data is the subject of this section which pursues the goal to fill the gap between mentioned areas and to develop a reasonable picture at $\mu \sim 1$ (say, $0.5<\mu<1.5$ ). There is good basis to think that the instability thresholds are located just in this region, and an attempt to estimate them seems very reasonable.

The overall picture is shown in Fig. 14 for the boxcar model. Exact solutions are known in this case, and two of them are presented in the figure by black lines. Corresponding e.f. are Legendre polynomials of 1 st and 2nd power, and e.v. starting from the point $\mu=\nu=0$ are plotted (trivial rigid mode is not discussed). Red lines present lower modes of Eq. (29) which formally refer to the case $\mu \ll 1$. However, it is seen that the approximate solution actually coincides with the exact one at $n=1$ and any $\mu$ [this fact has been explained after Eq. (31)], whereas good agreement is observed at $n=2$ and $\mu \lesssim 0.8$. The blue lines in Fig. 14 are e.v. of dipole and quadrupole modes $m=1$ and 2 [see Eq. (41)]. Again, there is very good agreement with exact solutions at $\mu \geqslant 0.8$, though formally Eq. (41) is valid only at $\mu \gg 1$. Hence, both considered models provide a satisfactory precision in the region of interest to us, though low- $\mu$ approximation works better at $\mu \lesssim 0.8$, and high- $\mu$ approximation-at $\mu \geq 0.8$.

Similarly, numerical results are presented in Fig. 15 for the parabolic model (32) using both approximations. It is seen that relative positions of red and blue lines are about the same here as in Fig. 14. Because exact solutions are unknown now, maximal incoherent betatron frequencies averaged on synchrotron phases are used instead. Modulation by synchrotron frequency is taken into account 


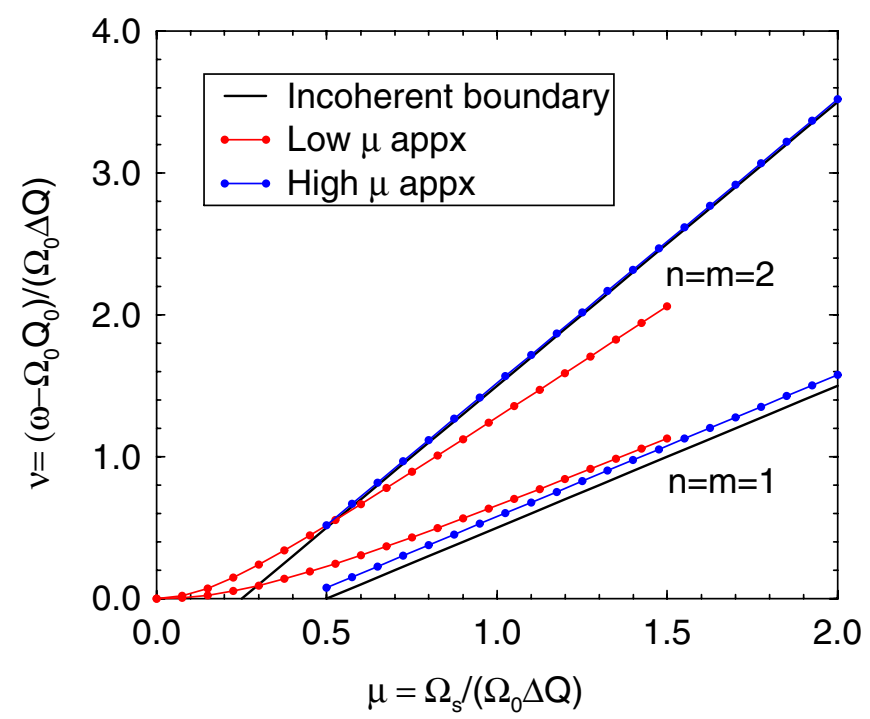

FIG. 15. (Color) Approximate eigenvalues and incoherent boundary of the parabolic bunch.

as well, so equations of the corresponding black lines are

$$
\nu=-0.5+m \mu \text {. }
$$

For each multipole, all incoherent frequencies are located below the corresponding line. Therefore, any eigenmode is stable (prone to Landau damping) if its e.v. are located below the line as well. According to high- $\mu$ approximation, both modes shown in Fig. 15 by blue circles are unstable. In contrast to this, the low- $\mu$ approximation predicts stability of the 2 nd mode at $\mu>0.5$ (red circles). However, this part of the curve must be ignored because the low- $\mu$ approach certainly is not applicable at more $\mu$. It allows one to establish the boundary between low- $\mu$ and

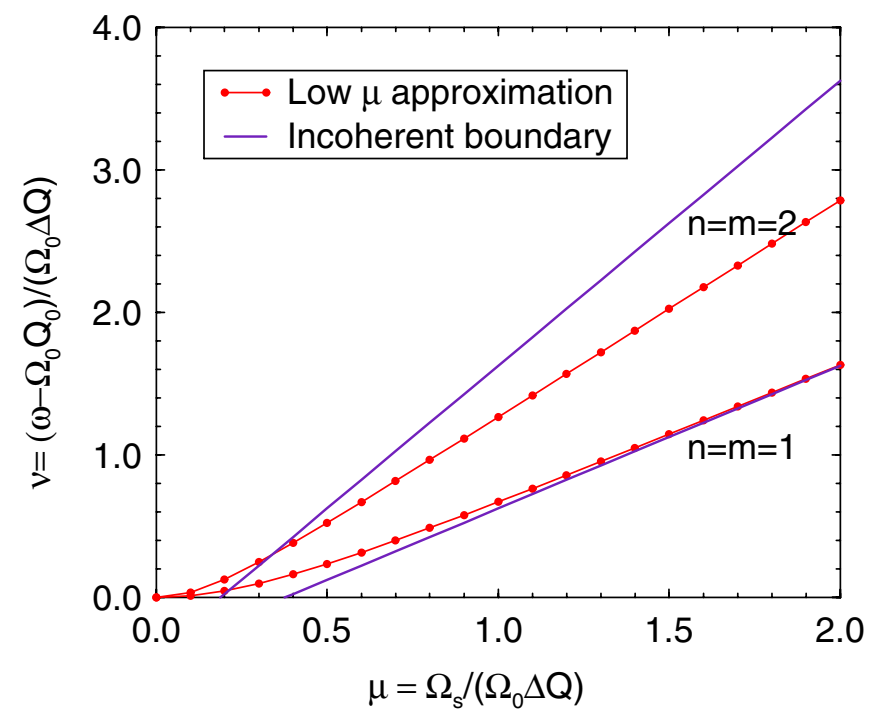

FIG. 16. (Color) Low- $\mu$ eigenvalues and incoherent boundaries of the "superparabolic" bunch. The continuous high- $\mu$ spectrum lies below the blue lines.

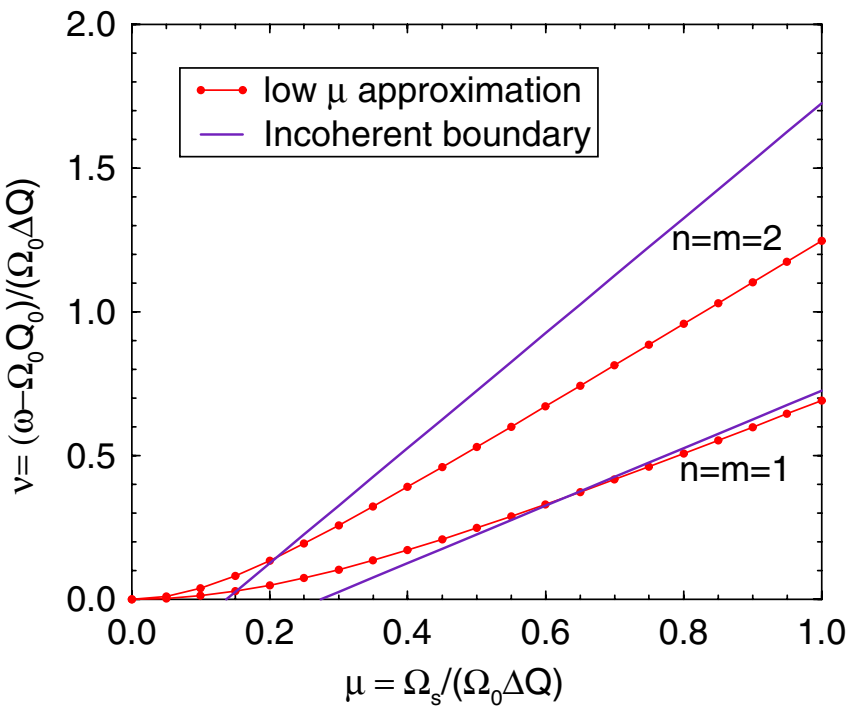

FIG. 17. (Color) The same as in Fig. 16 for the Gaussian bunch truncated on the $3 \sigma$ level.

TABLE II. Estimated instability thresholds.

\begin{tabular}{lll}
\hline \hline & $m=1$ & $m=2^{\mathrm{a}}$ \\
\hline S-parabolic & $\mu \simeq 1.5$ & $\mu \simeq 0.4$ \\
Gaussian & $\mu \simeq 0.6$ & $\mu \simeq 0.2$ \\
\hline \hline
\end{tabular}

${ }^{\text {a }}$ The higher modes are all the more stable.

high- $\mu$ approximations as $\mu \simeq 0.5-$ a result which is not very distinctive from the boxcar case.

Eigenvalues of superparabolic and truncated Gaussian bunches (33) and (34) are summarized in Figs. 16 and 17. High- $\mu$ approximation predicts that all their modes, except the rigid ones, are stable forming continuous spectra in the ranges

$$
\nu<-0.375+m \mu \quad \text { or } \quad \nu<-0.274+m \mu .
$$

Blue lines in the figures present highest e.v. of the spectra coinciding with incoherent boundaries. The low- $\mu$ approximation shown by the red lines asserts that these modes may be unstable at $\mu \ll 1$. However, they demonstrate relatively fast growth on the bunch tails at $\mu \geq 0.1$, and very fast (possibly infinite) growth at $\mu \gtrsim 0.5$ (see Figs. 4 and 6). Because similar behavior is a sign of Landau damping, one can estimate the instability thresholds as it is presented in Table II.

\section{CONCLUSION}

Transverse eigenmodes of a bunched beam are explored in this article at space charge dominated beam coupling impedance, that is at relatively slight wakefield. Synchrotron oscillations are taken into account, and any relation of synchrotron frequency to space charge tune shift 
is studied. The general equation for the eigenmodes of the bunch is derived, and its solutions are investigated in detail at linear synchrotron oscillations.

The main conclusion is that tune spread, produced by the space charge, brings a powerful Landau damping which suppresses most of the transverse modes. Without the wakefield, it formally appears as a continuum of singular eigenfunctions with real eigenfrequencies located inside the incoherent tunes range.

The rigid mode is the only solution which is never prone to this kind of damping. It does not depend on synchrotron oscillations at all, and in the simplest case (no effective chromaticity) manifests itself as transverse oscillations of the bunch as a whole-without bend or rotation. A traveling wave of transverse displacement is added at nonzero chromaticity.

Other modes are suppressed fully if synchrotron frequency exceeds space charge tune shift, and longitudinal distribution function has no abrupt boundaries. If the synchrotron frequency is lower, several modes other than the rigid one can overcome Landau damping being potentially unstable. A distinguishing characteristic of similar modes is a minor global dipole moment (zero without the chromaticity), and therefore a relatively weak expected long-term wakefield. In particular, the dipole mode of a truncated Gaussian bunch may be unstable at $\Omega_{s} \lesssim$ $0.6 \Omega_{0} \Delta Q(0)$, quadrupole one-at $\Omega_{s} \lesssim 0.2 \Omega_{0} \Delta Q(0)$, and the higher modes are more stable.

A great impact of dominated space charge impedance on instability threshold is commonly known for coasting beams, when the instability becomes possible if the space charge tune shift exceeds the tune spread. Real impedance is directly responsible for the instability and determines its growth rate, but it only slightly affects the threshold. A distinctive property of bunched beams is that the space charge not only brings coherent tune shift, but also creates incoherent tune spread because of variable bunch density.
When this takes place, Landau damping depends on the bunch shape being negligible in the boxcar bunch and suppressing almost all modes in smooth bunches like Gaussian.

Potentially unstable eigenfunctions can be used in the next steps for calculation of a wakefield which produces small additions to the eigenfrequencies, including the imaginary part, that is the instability growth rate. Global beam structure is essential at these calculations, determining characteristics of the collective modes. A detailed investigation of the collective motion based on the rigid mode oscillations is presented in Ref. [10]. In particular, it is shown that the growth rate of resistive wall instability could reach 3000/ sec in the Fermilab Recycler in frames of Project X, and $27 \mathrm{MHz}$ damper or chromaticity of about -7 could be required to suppress it. Without a doubt, other potentially unstable modes are not so dangerous because of a relatively low integral dipole moment of any bunch; however, these investigations are beyond the scope of the presented work.

[1] C. Pellegrini, Nuovo Cimento A 64, 447 (1969).

[2] M. Sands, Report No. SLAC TN-69-8, 1969.

[3] F. Sacherer, Report No. CERN-SI-BR-72-5, 1972.

[4] V. Balbekov, Zh. Tekh. Fiz. 46, 1470 (1976) [Sov. Phys. Tech. Phys. 21, 837 (1976)].

[5] M. Blaskiewicz, Phys. Rev. ST Accel. Beams 1, 044201 (1998).

[6] A. Burov, Phys. Rev. ST Accel. Beams 12, 044202 (2009); 12, 109901 (2009).

[7] V. Balbekov, Report No. Fermilab-FN-0783-AD, 2006.

[8] A. Chao, Physics of Collective Beam Instabilities in High Energy Accelerators (Wiley, New York, 1993), p. 172.

[9] L. Landau, J. Phys. USSR 10, 25 (1946).

[10] V. Balbekov, Report No. Fermilab-TM-2372-AD, 2007. 